\title{
Clinical evaluation and surveillance imaging of children with myelomeningocele and shunted hydrocephalus: a follow-up study
}

\author{
Jeremy S. Wetzel, MD, ${ }^{1}$ David P. Heaner, BS, ${ }^{2}$ Brandon C. Gabel, MD, ${ }^{2}$ \\ R. Shane Tubbs, PhD, PA-C, ${ }^{2}$ and Joshua J. Chern, MD, PhD',2 \\ 1'Department of Neurosurgery, Emory University School of Medicine, Atlanta; and 2Pediatric Neurosurgery Associates, Children's \\ Healthcare of Atlanta, Georgia
}

OBJECTIVE The majority of children with myelomeningocele undergo implantation of CSF shunts. The efficacy of adding surveillance imaging to clinical evaluation during routine follow-up as a means to minimize the hazard associated with future shunt failure has not been thoroughly studied.

METHODS A total of 300 spina bifida clinic visits during the calendar years between 2012 and 2016 were selected for this study (defined as the index clinic visit). Each index visit was preceded by a 6 -month period during which no shunt evaluation of any kind was performed. At the index clinic visit, all patients were evaluated by a neurosurgeon. Seventyfour patients underwent previously scheduled surveillance CT or shunt series scans in addition to clinical evaluation (surveillance imaging group), and 226 patients did not undergo surveillance imaging (clinical evaluation group). Subsequent unexpected events, defined as emergency department visits, caregiver-requested clinic visits, and shunt revision surgeries were reviewed. The timing and likelihood of an unexpected event in each of the 2 groups were compared using Cox proportional hazard survival analysis. The rate of shunt revision surgery in the follow-up period as well as the associated outcomes and rate of complications were analyzed.

RESULTS The clinical characteristics of the 2 groups were similar. In the clinical evaluation group, 4 of $226(1.8 \%)$ patients underwent shunt revision based on clinical findings during the index visit, compared to 8 of $74(10.8 \%)$ patients in the surveillance imaging group who underwent shunt revision based on clinical and imaging findings at that visit $(p<$ 0.05). In the subsequent follow-up period, there were 74 unexpected events resulting in 10 shunt revisions in the clinical evaluation group, for an event rate of $33 \%$ and operation rate of $13.5 \%$. In the surveillance imaging group there were 23 unexpected events resulting in 2 shunt revisions, for an event rate of $34.8 \%$ and an operation rate of $8.7 \%$; neither difference was statistically significant. The complication rate for shunt revision surgery was also not different between the groups.

CONCLUSIONS Obtaining predecided, routine surveillance imaging in children with myelomeningocele and shunted hydrocephalus resulted in more shunt revisions in asymptomatic patients. For patients who had negative results on surveillance imaging, the rate of shunt revision in the follow-up period was not significantly decreased compared to patients who underwent clinical examination only at the index visit.

https://thejns.org/doi/abs/10.3171/2018.7.PEDS1826

KEYWORDS myelomeningocele; shunted hydrocephalus; surveillance imaging

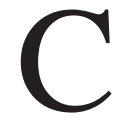
EREBROSPINAL fluid shunting procedures are some of the most common operations performed by neurosurgeons. Shunts fail for many reasons, and when failure occurs it can be life-threatening in some cases. Despite this concern, there is no consensus in the neurosurgical community about the appropriateness of surveillance imaging to detect shunts that may be malfunctioning prior to the onset of overt symptomatology. ${ }^{4}$ Some neurosurgeons follow patients clinically and order imaging only if there is clinical concern for shunt failure, whereas others order surveillance imaging at predetermined intervals to detect potential malfunctions before symptoms become apparent. One difficulty in designing such a study is the heterogeneity inherent to the different populations of pa-

ABBREVIATIONS ED = emergency department; EVD = external ventricular drain; IVH = intraventricular hemorrhage; $\mathrm{MMC}=$ myelomeningocele.

SUBMITTED January 13, 2018. ACCEPTED July 24, 2018.

INCLUDE WHEN CITING Published online October 19, 2018; DOI: 10.3171/2018.7.PEDS1826. 
tients with shunted hydrocephalus. The various etiologies of hydrocephalus are likely to follow different patterns of malfunction and clinical presentation, and therefore warrant a distinct approach to management. ${ }^{4}$

Of all populations of patients with shunted hydrocephalus, the myelomeningocele (MMC) population is relatively homogeneous and, given the need for multidisciplinary specialized care, they tend to have reliable follow-up patterns, making it an ideal population for study. In a typical multidisciplinary spina bifida specialty clinic, every patient with a CSF shunt is evaluated by a neurosurgeon, but not every patient has a surveillance $C T$ or shunt series scan at every visit. In a previously published study based on patients in a single location, we had found that surveillance imaging failed to decrease the likelihood that future shunt revisions would be needed despite an increased number of operations in patients with asymptomatic ventricle enlargement. ${ }^{2}$ We are now using the same study criteria in a second location with the goal of determining if surveillance imaging reduces emergency department (ED) visits, follow-up requests, frequency of revision, mortality, and morbidity related to shunt malfunction.

\section{Methods}

\section{The Care of Patients With MMC}

At Children's Healthcare of Atlanta, the largest children's hospital in the state of Georgia, patients with MMC receive care from a multidisciplinary team of neurosurgeons, orthopedic surgeons, urologists, pediatricians, rehabilitation physicians, physical therapists, and a dedicated full-time clinical coordinator in a specialty clinic setting. A team of 5 board-certified pediatric neurosurgeons provided neurosurgical care to these patients during the study period. Each patient was assigned to 1 of the 5 neurosurgeons to maintain continuity of care. There was no consensus practice standard regarding the use of scheduled surveillance imaging among the surgeons in the group. Whether or not predetermined surveillance imaging was obtained was entirely according to surgeon preference. When surveillance imaging scans were obtained, they generally occurred at 6-month intervals in patients between 1 and 3 years of age, annually in patients between 3 and 7 years of age, and biannually after that, so as to coincide with the interval of office visits. Whereas the follow-up intervals were generally shorter in younger patients, the tendency to obtain surveillance imaging was lower in general, given the ability to follow these patients' open fontanelles and head circumference measurements. In the case of ventriculoatrial shunts in a growing child, it is fairly standard practice to obtain a chest radiograph every 1-2 years, but this is ordered independent of any brain imaging in most cases, and is done to ensure that the catheter tip remains at the ideal location.

It was the practice of all surgeons in the group to revise the shunts in patients with MMC who were asymptomatic but who had notable ventricular enlargement on imaging when compared to prior scans. Although there was no consensus regarding the percent change in ventricular caliber in asymptomatic patients necessary to prompt intervention, minimal changes were likely to be observed and significant changes were explored. This was managed on a case-by-case basis and the change in ventricular size was not quantified.

\section{Patient Selection}

Patients with MMC with shunted hydrocephalus who had a scheduled visit to the spina bifida specialty clinic in the calendar years of 2012 and 2016 (index visit) were considered for this study. All of the patients had already attended the follow-up visit or had experienced a subsequent event of interest. The period between the index clinic visit and the subsequent scheduled visit or an event was defined as the follow-up period.

Eighty patients had only 1 visit between 1/1/2012 and $12 / 31 / 2016$, and these visits were excluded. Twenty-three of these 80 patients were transitioned to the care of neurosurgeons who treat adult patients. One hundred seven visits were excluded because the patient had been evaluated by the neurosurgery service within 6 months preceding the index visit. This included any clinic visits, ED visits, or hospital admissions. Patients were excluded if they had been hospitalized by other services even if the neurosurgery service was not formally consulted to participate in their care. Patients were excluded if there had been a preceding shunt exploration/revision within 6 months. Visits made by infants younger than 6 months of age were excluded because they did not have a 6-month interval free of neurosurgical evaluation (93 visits). Patients whose care was transferred to Children's Healthcare of Atlanta and came to the spina bifida clinic for the first time were excluded, because their past history was not always complete.

A total of 300 clinical visits fulfilled the above inclusion and exclusion criteria. In summary, these were visits made by patients with MMC and shunted hydrocephalus who had had an unremarkable clinical course in the 6-month period leading up to the index visit and a documented follow-up visit. For these patients, some would have undergone surveillance imaging on the day of the index clinic visit based on clinical orders placed during the previous visit. Seventy-four patients (24.7\%) had routine surveillance imaging in addition to clinical examination, and the remaining $226(75.3 \%)$ had clinical examination alone.

\section{Statistical Analysis}

Statistical analysis was performed using SPSS software (SPSS, Inc.). The event-free survival of the CSF shunt was defined as the length of time between the index clinic visit and the specified "end" events. The patients were dichotomized into the clinical evaluation group versus the surveillance imaging group. The end events were an ED visit with shunt-related complaint, a request from a caregiver to have the patient examined in the neurosurgery clinic prior to the previously scheduled follow-up visit, or a shunt operation. If neither of the end events occurred before the patient attended the follow-up visit, the CSF shunt was considered to function adequately and the case was considered event-free in the survival analysis. In the analysis, the use of surveillance imaging was defined as 
a categorical predictor (patients who did or did not undergo surveillance CT or shunt series). Cox proportional hazard analysis was then used to examine whether or not the CSF shunt event-free survival was predicted by the use of surveillance imaging. The occurrence of surgical complications from shunt revision/exploration in the 2 groups was compared using a contingency table. The rate of shunt revision based on findings at the index visit, either radiographic or clinical examination alone, was compared using a standard chi-square test. Probability values less than 0.05 were considered statistically significant.

\section{Comparison of Results to Prior Study}

Because it is critical in research to validate substantial and clinically meaningful findings in both space and time, we have chosen to replicate the methods of a previously published study by the principal investigator (J.J.C.). Identical methods were used for both studies, and the results and implications are discussed further in the ensuing sections.

\section{Results}

\section{Events at the Index Clinic Visit}

The average age in the clinical evaluation group and the surveillance imaging group was 10.4 (SD 6.1) and 8.0 (SD 6.1) years, respectively. The average length of follow-up in the 2 groups was 9.9 (SD 5.9) and 10.4 (SD 7.0) months, respectively.

In the clinical evaluation group, 4 of 226 patients (1.8\%) underwent shunt revision surgery based on clinical evidence of shunt malfunction (Table 1). In all cases imaging studies were obtained after examination based on clinical concerns. The patient in case 10 presented with headaches and bilateral upper-extremity paresthesias. Brain and Cspine MRI showed stable ventricular size but enlargement of a known cervical syrinx. At surgery, both proximal and distal malfunctions were discovered. Two patients had mild headaches on evaluation and were found to have interval ventricular enlargement on CT. Both patients (cases 11 and 12) were found to have shunt malfunctions at surgery. The patient in case 9 presented to clinic with a palpable disconnection in her cervical shunt tubing, and imaging confirmed fractured distal tubing, although ventricular size was stable on CT. All patients in this group had positive findings during shunt revision surgery and none had complications related to surgery.

Of the 74 patients who had surveillance imaging and clinical evaluation, 8 patients $(10.8 \%)$ underwent surgical intervention as a result of imaging and clinical findings (Table 1). Compared to the clinical examination group, this difference was statistically significant $(\mathrm{p}<0.05)$. Four patients were asymptomatic, with enlarged ventricles seen on CT scans (cases 2, 3,6, and 8). All 4 patients underwent shunt revision surgeries, and an obstruction of CSF flow at some point along the shunt system was documented in all 4 cases (i.e., proximal, distal, valve, or a combination thereof). One patient had a palpable fluid collection along the distal shunt tubing and was found to have a distal malfunction (case 1). The final 3 patients were thought to be in need of distal shunt lengthening based on the shunt series,
TABLE 1. Summary of clinical events after the initial clinic visit and in the follow-up period in $\mathbf{3 0 0}$ patients with shunt-treated hydrocephalus

\begin{tabular}{lccc}
\hline \multicolumn{1}{c}{ Clinical Events } & $\begin{array}{c}\text { Clinical } \\
\text { Evaluation } \\
\text { Only }\end{array}$ & $\begin{array}{c}\text { Clinical \& } \\
\text { Radiological } \\
\text { Evaluation }\end{array}$ & $\begin{array}{c}\text { p Value } \\
\text { Where } \\
\text { Relevant }\end{array}$ \\
\hline $\begin{array}{c}\text { No. of patients } \\
\text { No. of ops due to findings at } \\
\text { initial clinic visit }\end{array}$ & $4(1.8 \%)$ & $8(10.8 \%)$ & 0.00057 \\
\hline $\begin{array}{l}\text { Shunts deemed to be functional } \\
\text { at initial clinic visit }\end{array}$ & 222 & 66 & \\
\hline $\begin{array}{l}\text { Events in FU period } \\
\text { ED visits (no. of ops) }\end{array}$ & $52(9)$ & $16(1)$ & 0.890 \\
\hline $\begin{array}{l}\% \text { of functional shunts at } \\
\text { 1st visit }\end{array}$ & $23 \%$ & $24 \%$ & \\
\hline $\begin{array}{l}\text { Caregiver-requested visits } \\
\text { (no. of ops) }\end{array}$ & $22(1)$ & $7(1)$ & 0.869 \\
\hline $\begin{array}{l}\% \text { of functional shunts at } \\
\text { 1st visit }\end{array}$ & $10 \%$ & $11 \%$ & \\
\hline $\begin{array}{l}\text { Total no. of shunt ops during } \\
\text { FU period (total revision } \\
\text { rate) }\end{array}$ & $10(4.5 \%)$ & $2(3 \%)$ & 0.819 \\
\hline
\end{tabular}

$\mathrm{FU}=$ follow-up.

and showed no evidence of shunt malfunction at surgery (cases 4, 5, and 7).

\section{Unexpected Events in the Follow-Up Period}

Patients who underwent shunt revision/exploration due to findings at the index clinic visit were excluded from this part of the analysis. Therefore, the event-free survival analysis included only those patients who were deemed to have a functioning shunt based on clinical alone $(\mathrm{n}=$ 222 ) or clinical and radiological $(n=66)$ findings (Table 1 ). The relevant clinical events (ED visits and caregiverrequested visits for shunt-related complaints) in the follow-up periods of the 2 groups are summarized in Table 1. The 2 CSF shunt event-free survival curves were then plotted and compared using the Cox proportional hazards method (Fig. 1).

In the clinical examination group, 74 of 222 patients experienced an unexpected event. There were $52 \mathrm{ED}$ visits and 22 requested clinic visits, for a total event rate of $33 \%$ in this group. In the surveillance imaging group, 23 of 66 patients had an unexpected event (7 ED visits and 16 requested clinic visits) for a total event rate of $34.8 \%$. This difference in the total event rate between the 2 groups did not reach statistical significance $(p=0.82)$. The hazard ratio of experiencing an event if a patient was in the surveillance imaging group was 1.0455 (95\% CI 0.7159-1.5268; $\mathrm{p}=0.82$ ).

The number of shunt surgeries for the 2 groups of patients is summarized in Table 1 . Of the 222 patients in the clinical examination group who were deemed to have a functional shunt at the end of the index visit, 10 required shunt revisions, for a total revision rate of $4.5 \%$. This is compared to 2 of the 66 patients in the surveillance imag- 


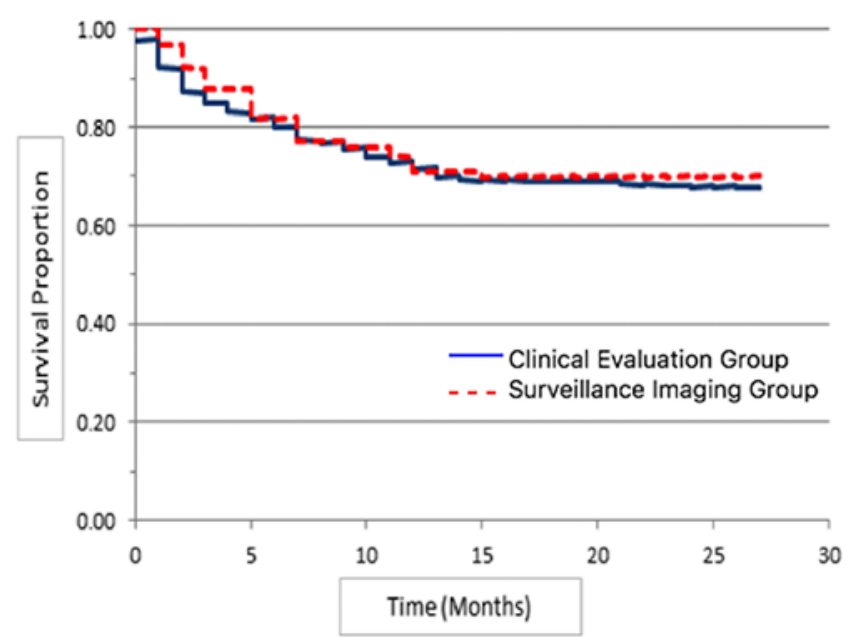

FIG. 1. Event-free survival of a CSF shunt after the index clinic visit. Figure is available in color online only.

ing group who required a shunt revision, for a total revision rate of $3.0 \%$. There was no statistically significant difference in revision rates between the 2 groups $(p=0.60)$.

\section{Surgical Complications}

The details of clinical presentation, surgical findings, and complications of shunt revision/explorations are summarized in Tables 2 and 3 . In the 12 patients who underwent surgical exploration and shunt revision based on findings in the index clinic visit, there were no complications (Table 2).

Another 12 patients underwent shunt revision in the follow-up period. Only 1 patient experienced a complication related to surgery (case 2, Table 3 ). This patient suf- fered a minor intraventricular hemorrhage (IVH) caused by replacement of the proximal shunt catheter, requiring external ventricular drain (EVD) placement to clear the IVH. The shunt was replaced 6 days later and patient was discharged having suffered no significant morbidity as a result of the complication.

\section{Discussion}

\section{Study Design}

One of the primary difficulties in studying the utility of surveillance imaging in patients with shunted hydrocephalus is the heterogeneity of these patient populations. Shunted hydrocephalus of various etiologies may differ in the failure pattern; each etiology may be uniquely associated with other disabilities that pose challenges in diagnosing shunt malfunction. ${ }^{4}$ For example, posthemorrhagic hydrocephalus associated with prematurity and that caused by a third-ventricle neoplasm may both be treated with a CSF shunt, but the differences in underlying pathology and patient characteristics do not allow for direct comparisons of the shunt management strategy in the 2 groups.

Patients with MMC comprise one of the largest groups of patients treated with CSF shunts. They are relatively homogeneous in terms of the pathophysiology and also shunt failure patterns. More often than not they access medical care through specialty clinics, which was popularized in the 1970s and dramatically improved outcome. ${ }^{1,3}$ Because of these dedicated clinics, patients tend to receive all of their medical care at a single institution. This minimizes the number of patients who are lost to follow-up. Last, these patients and their caregivers are often not strangers to shunt malfunctions, which implies a certain degree of vigilance and familiarity with the presentation of shunt malfunction. Although these characteristics are essential

TABLE 2. Clinical outcomes in 12 patients undergoing shunt exploration and revision based on findings at the initial clinic visit

\begin{tabular}{|c|c|c|c|c|c|c|}
\hline $\begin{array}{l}\text { Case } \\
\text { No. }\end{array}$ & $\begin{array}{l}\text { Age } \\
\text { (yrs) }\end{array}$ & $\begin{array}{c}\text { Surveillance } \\
\text { CT }\end{array}$ & Reason for Revision & $\begin{array}{l}\text { LOS } \\
\text { (days) }\end{array}$ & Shunt Exploration Findings & $\begin{array}{c}\text { Complications } \\
\text { w/in } 6 \text { Mos }\end{array}$ \\
\hline 1 & 1 & Yes & Swelling along shunt tract & 3 & Distal obstruction & None \\
\hline 2 & 7 & Yes & Asymptomatic enlargement of ventricles & 6 & Proximal obstruction & None \\
\hline 3 & 7 & Yes & Asymptomatic enlargement of ventricles & 3 & Proximal \& distal disconnection from valve & None \\
\hline 4 & 7 & Yes & Distal catheter lengthening & 7 & Negative & None \\
\hline 5 & 12 & Yes & Distal catheter lengthening & 7 & Negative & None \\
\hline $6^{*}$ & 12 & Yes & Asymptomatic enlargement of ventricles & 17 & Distal obstruction & None \\
\hline 7 & 13 & Yes & Distal catheter lengthening & 6 & Negative & None \\
\hline 8 & 17 & Yes & Asymptomatic enlargement of ventricles & 2 & Proximal obstruction & None \\
\hline 9 & 13 & No & $\begin{array}{l}\text { Palpable break in shunt tubing: stable ventricles, } \\
\text { fractured catheter }\end{array}$ & 3 & Fractured shunt tubing & None \\
\hline 10 & 14 & No & $\begin{array}{l}\text { HAs, hand paresthesias; incr in size of cervical syrinx } \\
\text { but stable ventricles }\end{array}$ & 9 & Proximal \& distal obstruction & None \\
\hline 11 & 17 & No & HAs, enlargement of ventricles & 2 & Distal obstruction & None \\
\hline 12 & 11 & No & HAs, enlargement of ventricles, preperitoneal catheter & 2 & Distal catheter preperitoneal & None \\
\hline
\end{tabular}

$\mathrm{HA}=$ headache; incr = increase; LOS = length of stay.

These 12 patients were seen in the office for the initial visit and had some problem that was acted upon, whether or not a scan was obtained.

* Patient in case 6 had a grossly infected ankle treated with orthopedic surgery and prolonged antibiotic administration, along with ventriculoatrial shunt revision. 
TABLE 3. Clinical outcomes in 12 patients undergoing shunt exploration and revision in the follow-up period

\begin{tabular}{|c|c|c|c|c|c|c|}
\hline $\begin{array}{l}\text { Case } \\
\text { No. }\end{array}$ & $\begin{array}{l}\text { Age } \\
\text { (yrs) }\end{array}$ & $\begin{array}{l}\text { Surveillance } \\
\text { CT }\end{array}$ & Reason for Revision & $\begin{array}{l}\text { LOS } \\
\text { (days) }\end{array}$ & Shunt Exploration Findings & $\begin{array}{l}\text { Intraop \& 6-Mo } \\
\text { Complications }\end{array}$ \\
\hline 1 & 3 & No & Symptomatic, enlargement in ventricular size, presented to ED & 4 & Proximal obstruction & None \\
\hline $2^{*}$ & 5 & No & $\begin{array}{l}\text { Symptomatic, enlargement in ventricular size, presented to } \\
\text { clinic }\end{array}$ & 17 & Proximal obstruction & $\begin{array}{l}2 \text { EVD placements } \\
\text { followed by } \\
\text { VPS reinsertion }\end{array}$ \\
\hline 3 & 6 & No & Symptomatic, enlargement in ventricular size, presented to ED & 2 & Distal obstruction & None \\
\hline 4 & 7 & No & Symptomatic, enlargement in ventricular size, presented to ED & 2 & Proximal obstruction & None \\
\hline 5 & 7 & No & Symptomatic, enlargement in ventricular size, presented to ED & 3 & Holes in distal catheter tubing & None \\
\hline 6 & 13 & No & $\begin{array}{l}\text { Symptomatic, enlargement in ventricular size, fractured cath- } \\
\text { eter, presented to ED }\end{array}$ & 2 & $\begin{array}{l}\text { Proximal disconnection with } \\
\text { multiple holes in tubing }\end{array}$ & None \\
\hline 7 & 15 & No & $\begin{array}{l}\text { Symptomatic, enlargement of ventricular size, ventricular } \\
\text { dislodgment of catheter, presented to ED }\end{array}$ & 2 & $\begin{array}{l}\text { Ventricular dislodgment of } \\
\text { catheter }\end{array}$ & None \\
\hline 8 & 18 & No & $\begin{array}{l}\text { Symptomatic, enlargement of ventricular size, ventricular } \\
\text { dislodgment of catheter, presented to ED }\end{array}$ & 2 & Proximal obstruction & None \\
\hline 9 & 8 & No & Symptomatic, enlargement in ventricular size, presented to ED & 7 & Proximal obstruction & None \\
\hline 10 & 16 & No & Symptomatic, presented to ED & 6 & Distal obstruction & None \\
\hline 11 & 11 & Yes & Torticollis, presented to clinic & 2 & Negative & None \\
\hline 12 & 15 & Yes & Symptomatic, enlargement in ventricular size, presented to ED & 2 & Proximal obstruction & None \\
\hline
\end{tabular}

VPS = ventriculoperitoneal shunt.

These 12 patients were determined to have working shunts at their initial visit (so the patients in Table 2 were excluded from this part of the analysis), but they experienced events in the follow-up period such as presenting to the ED with symptoms.

* An IVH occurred after VPS revision followed by the first EVD placement. Rehemorrhaging occurred, resulting in a second EVD placement. Shunt was reinserted 6 days later.

enablers of the current study, they also limit the generalizability of the results. With this in mind, we hope that the results of this investigation will help direct the future study of the management of hydrocephalus in other populations.

It should be noted that the current investigation was undertaken to see whether results from a prior study based in Birmingham, Alabama, could be replicated. ${ }^{2}$ Identical study design and patient selections were used in both studies, and the senior author (J.J.C.) is the principal investigator for both studies. Conducting identical studies in different sites may potentially mitigate some of the bias introduced by the clinicians involved and by the distinct healthcare delivery systems. It also includes a larger number of patients so that smaller differences may be detected.

\section{Comparison of Results}

In the current study, in patients who were deemed to have functional shunts, whether by clinical examination alone or with the addition of surveillance imaging, there was no difference in the incidence of ED visits, caregiver-requested clinic visits, rate of shunt surgery, or rate of surgical complications in the follow-up period. In other words, aside from detecting asymptomatic changes in ventricular caliber or shunt tubing disconnections, the additional expense and radiation exposure of surveillance imaging did not add any benefit to the overall care of this patient population.

Because our current study follows the identical design of a previous study based in Birmingham, Alabama, results from both studies were placed side-by-side in Table
4 for comparison. Patient populations were similar in age ( 9.5 vs 10.0 years, current vs previous study) and length of follow-up (10.3 vs 9.9 months, current vs previous study). These patients were under the care of 10 different boardcertified pediatric neurosurgeons between the years of 2008 and 2009 (Birmingham) and 2012 and 2016 (Atlanta). Neither locality had an explicitly stated policy regarding to the use of surveillance imaging, but in both places less than $50 \%$ of patients underwent previously scheduled surveillance imaging at the time of the index clinic visit. Reaching statistical significance in both places, a significant number of patients underwent shunt revisions based on findings from the surveillance imaging. However, does this preemptive elimination of potential shunt malfunction improve the odds of shunt survival until the next clinic visit? The data from both studies suggest that the difference in shunt survival is likely to be minimal. When the results of both study groups, from Atlanta and Birmingham, are pooled together, the shunt revision rate during the follow-up period in the clinical evaluation group was $4.9 \%((10+12) /(222+228))$ and in the surveillance imaging group it was $4.5 \%((2+8) /(66+155)) ; \mathrm{p}=0.84$.

\section{Surgeries for Asymptomatic Shunt Malfunction}

It is important to point out that this study does not directly argue for or against revisions for asymptomatic ventriculomegaly in a patient with a shunt. The utility of shunt revision in this context is not only to decrease morbidity and mortality related to acute shunt malfunction but also to protect against subtle neurocognitive deterioration. ${ }^{5,6}$ Our previous and current studies do not address cogni- 
TABLE 4. Comparison of results from Birmingham, Alabama, and Atlanta, Georgia

\begin{tabular}{|c|c|c|c|c|c|c|}
\hline \multirow[b]{2}{*}{ Clinical Events } & \multicolumn{3}{|c|}{ Atlanta, GA (current study) } & \multicolumn{3}{|c|}{ Birmingham, $\mathrm{AL}$} \\
\hline & $\begin{array}{c}\text { Clinical } \\
\text { Evaluation } \\
\text { Only }\end{array}$ & $\begin{array}{c}\text { Clinical \& } \\
\text { Radiological } \\
\text { Evaluation }\end{array}$ & $\begin{array}{l}\text { p Value } \\
\text { Where } \\
\text { Relevant }\end{array}$ & $\begin{array}{l}\text { Clinical } \\
\text { Evaluation } \\
\text { Only }\end{array}$ & $\begin{array}{c}\text { Clinical \& } \\
\text { Radiological } \\
\text { Evaluation }\end{array}$ & $\begin{array}{l}\text { p Value } \\
\text { Where } \\
\text { Relevant }\end{array}$ \\
\hline No. of patients & 226 & 74 & & 230 & 166 & \\
\hline No. of ops due to findings at initial clinic visit & $4(1.8 \%)$ & $8(10.8 \%)$ & 0.00057 & $2(0.9 \%)$ & $11(6.6 \%)$ & 0.0015 \\
\hline Shunts deemed to be functional at initial clinic visit & 222 & 66 & & 228 & 155 & \\
\hline \multicolumn{7}{|l|}{ Events in FU period } \\
\hline ED visits (no. of ops) & $52(9)$ & $16(1)$ & 0.890 & $27(7)$ & $15(7)$ & 0.506 \\
\hline$\%$ of functional shunts at 1 st visit & $23 \%$ & $24 \%$ & & $12 \%$ & $10 \%$ & \\
\hline Caregiver-requested visits (no. of ops) & $22(1)$ & $7(1)$ & 0.869 & $25(5)$ & $9(1)$ & 0.0815 \\
\hline$\%$ of functional shunts at 1 st visit & $10 \%$ & $11 \%$ & & $11 \%$ & $6 \%$ & \\
\hline Total no. of shunt ops during FU period (total revision rate) & $10(4.5 \%)$ & $2(3.0 \%)$ & 0.616 & $12(5.3 \%)$ & $8(5.2 \%)$ & 0.965 \\
\hline
\end{tabular}

tive or functional outcome. A prospective randomized trial with serial neurocognitive testing would be necessary to provide the answer. However, before randomizing patients to an imaging or no-imaging group, there needs to be a study showing that the severity and likelihood of harm is equivalent in the 2 groups. The combined results of these 2 studies gave such an assurance.

\section{Conclusions}

Based on these findings and those of the previous study by Chern et al., routine surveillance imaging in patients with MMC and shunted hydrocephalus who have not required hospital admission or other special attention to their shunt in the preceding 6 months is unlikely to be of benefit. However, this decision should be made on a case-bycase basis. Although our objective was to use this followup study to further validate the results of the prior study by using identical methodology separated by both space and time, there are inherent limitations of these retrospective study designs. Additional studies are needed, ideally of prospective, randomized, multicenter design, to clarify the issue of surveillance imaging in other populations of patients with shunted hydrocephalus.

\section{References}

1. Beuriat PA, Poirot I, Hameury F, Szathmari A, Rousselle C, Sabatier I, et al: Postnatal management of myelomeningocele: outcome with a multidisciplinary team experience. World Neurosurg 110:e24-e31, 2018

2. Chern JJ, Muhleman M, Tubbs RS, Miller JH, Johnston JM, Wellons JC III, et al: Clinical evaluation and surveillance imaging in children with spina bifida aperta and shunt-treated hydrocephalus. J Neurosurg Pediatr 9:621-626, 2012
3. Fletcher JM, Brei TJ: Introduction: spina bifida-a multidisciplinary perspective. Dev Disabil Res Rev 16:1-5, 2010

4. Iskandar BJ, McLaughlin C, Mapstone TB, Grabb PA, Oakes WJ: Pitfalls in the diagnosis of ventricular shunt dysfunction: radiology reports and ventricular size. Pediatrics 101:10311036, 1998

5. McNatt SA, Kim A, Hohuan D, Krieger M, McComb JG: Pediatric shunt malfunction without ventricular dilatation. Pediatr Neurosurg 44:128-132, 2008

6. Vinchon M, Fichten A, Delestret I, Dhellemmes P: Shunt revision for asymptomatic failure: surgical and clinical results. Neurosurgery 52:347-356, 2003

\section{Disclosures}

The authors report no conflict of interest concerning the materials or methods used in this study or the findings specified in this paper.

\section{Author Contributions}

Conception and design: Chern. Acquisition of data: Chern. Analysis and interpretation of data: Wetzel, Heaner, Chern. Drafting the article: Wetzel, Heaner, Gabel, Chern. Critically revising the article: all authors. Reviewed submitted version of manuscript: all authors. Approved the final version of the manuscript on behalf of all authors: Wetzel. Statistical analysis: Wetzel, Heaner, Tubbs, Chern.

\section{Correspondence}

Jeremy S. Wetzel: Emory University School of Medicine, Atlanta, GA.jwetzel@emory.edu. 